\title{
The Phi-Bot: A Robot Controlled by a Slime Mould
}

\author{
Soichiro Tsuda ${ }^{1}$, Stefan Artmann ${ }^{2}$ \\ Klaus-Peter Zauner ${ }^{1}$ \\ ${ }^{1}$ School of Electronics and Computer Science \\ University of Southampton, SO17 1BJ, United Kingdom \\ ${ }^{2}$ Frege Centre for Structural Sciences, Friedrich-Schiller-University, \\ Zwätzengasse 9, 07737 Jena, Germany \\ st07v@ecs.soton.ac.uk
}

December 22, 2008

\section{Introduction}

Information processing in natural systems radically differs from current information technology. This difference is particularly apparent in the area of robotics, where both organisms and artificial devices face a similar challenge: the need to act in real time in a complex environment and to do so with computing resources severely limited by their size and power consumption. Biological systems evolved enviable computing capabilities to cope with noisy and harsh environments and to compete with rivalling life forms. Information processing in biological systems, from single-cell organisms to brains, directly utilises the physical and chemical processes of cellular and intracellular dynamics, whereas that in artificial systems is in principle independent of any physical implementation. The formidable gap between artificial and natural systems in terms of information processing capability [1] motivates research into biological modes of information processing. Hybrid artifacts, for example, try to overcome the theoretic and physical limits of information processing in solid-state realisations of digital von Neumann machines by exploiting the self-organisation of naturally evolved systems in engineered environments $[2,3]$.

This chapter presents a particular unconventional computing system, the $\Phi$ bot, whose control is based on the behaviour of the true slime mould Physarum polycephalum. The second section gives a short introduction to the informationprocessing capabilities of this organism. The third section describes the two generations of the $\Phi$-bot built so far. To discuss information-theoretic aspects of this robot it is useful to sketch the concept of bounded computability that 
relates generic traits of information-processing systems with specific physicochemical constraints on the realisation of such systems in different classes of computational media. This is done in the fourth section. The concluding section gives an outlook on engineering as well as foundational issues that will be important for the future development of the $\Phi$-bot.

\section{Physarum polycephalum as Information Pro- cessor}

The plasmodium of the true slime mould, Physarum polycephalum (Fig. 1), is an amoeba-like unicellular organism, whose body size ranges from several hundred microns to a radius of more than one meter. Despite its large size, the single cell acts as an integrated organism and is known for its distributed information processing.

A plasmodial cell of Physarum polycephalum consists of an ectoplasm tube that encloses an endoplasmic core. The former is a gel membrane layer, while the latter is a more fluid state of the protoplasm [4]. In the ectoplasm tube, cytoplasmic actomyosin periodically aggregates to form sheet-like structures and then unravels into fibrils. These structural changes create a hydrostatic pressure gradient within the cell, and eventually give rise to a flow of ectoplasm shuttling from one location in the cell to other parts of the cell and back. If a cell is not stimulated by any external stimuli, this contractile rhythm is synchronised throughout the cell. However, when a local part of a cell is exposed to an external stimulus such as food or white light, it leads to desynchronisation of the rhythm. The frequency of the oscillating rhythm at the stimulated part increases if it is an attractive stimulus, and decreases if it is repulsive. Such local frequency change affects oscillations of other parts through protoplasmic streaming and forms a spatial phase pattern in the cell $[5,6,7]$. The emerging global phase pattern eventually determines the direction of migration, i.e., the behaviour of the organism [8].

This mode of information processing affords scalability to the plasmodium. As long as the plasmodium is able to form the phase gradient of the contractile oscillation rhythm within its single-cell body, it reacts to various external stimuli in the same fashion no matter how large it grows. Central to this sizeinvariant behaviour is the spatial phase pattern of the oscillation rhythm formed within a cell. It emerges from the interaction of the intracellular dynamics of the plasmodium and the environment triggered by a contact with an external stimulus and the plasmodium. Several theoretical models have been proposed to explain the behaviour $[9,10]$ based on the theory of positional information [11]. Takamatsu, taking a systems perspective, constructed coupled nonlinear oscillator systems from the Physarum cell by controlling the cell shape using microfabricated structures $[12,13]$.

The distributed information processing that arises from the integration of oscillatory patterns in the cell enables the plasmodium to optimise food gathering 
strategies $[14,15]$.

It is interesting to note that the information processing in the cell can access information about past states. Nakagaki and his colleagues found if exposed to periodic environmental changes the plasmodium is able to anticipate the next change by changing its behaviour at the time a periodic change was next due to occur; the memory persisted over several hours [16].

\section{Cellular Robot Control}

The information-processing abilities of the plasmodium described above, together with the relative ease with which plamodial cells can be manipulated, facilitates the use of this simple organism in hybrid systems. Using the plasmodial cell may in the future provide adaptive behaviour in engineered systems. Robot control will be a good test bed for exploring this possibility. For this purpose, the contractile oscillation dynamics of the cell was employed to control robots. A Physarum plasmodium integrated into a robot is coupled to the robot's environment via bi-directional interfaces. External signals received by the robot are transferred to stimulating signals to the Physarum cell as robot brain, and the reaction of the cell is transferred back to the robot and interpreted as as a source of control signals for the robot. In the following subsections we will present our cellular robot approach, the $\Phi$-bot project, towards life-like autonomous robots.

\subsection{The first generation of the $\Phi$-bot: Tethered robot de- sign}

The Physarum-controlled robot consists of three components: a Physarum cell, a hexapod robot, and interfaces between them (Fig. 2).

\section{The Physarum circuit}

A Physarum plasmodium is confined in a star shape which consists of six $1.5 \mathrm{~mm}$ diameter circular wells and six $0.4 \mathrm{~mm}$ width channels connecting the circles at the centre (Fig. 2A). The plasmodium is guided to assume the starshape on a $1.5 \%$ agar gel by a negative plastic mask with a star-shape cutout. The plasmodium preferentially grows on a moist surface rather than a dry one so that it can absorb water from it. Accordingly, a Physarum plasmodium grows on the exposed agar surface and eventually takes on a star-shape. We refer to this shape-fixed Physarum cell as "Physarum circuit". It is known that the part of the plasmodium within a $1.5 \mathrm{~mm}$ diameter well shows a synchronised oscillation [12]. The formation and collapse of actomyosin structures within the cell pump the protoplasm through ectoplasm tubes in the channels of the mask.

The part of the plasmodium inside a well functions as a single oscillator, referred to as "Physarum oscillator", and is connected to the other oscillators (wells) through tubes in the channel and therefore the whole star-shaped cell can be viewed as a coupled nonlinear oscillator system [12].

The hexapod robot platform 
A hexapod robot is equipped with six leg actuators and six light sensors for omnidirectional behaviour and sensing (Fig. 2B). Each leg and sensor is designed to correspond to a Physarum oscillator so that the Physarum circuit can control the robot and interface with the robot's environment.

Each leg is driven by one of six independent servos (Fig. 2C), and either pushes or drags the robot body depending on the direction of its swing motion. Although each leg has only a single degree of freedom, in combination they afford numerous gate patterns and provide excellent stability. For example, anti-phase motion between opposite legs (para-position) yields directed motion of the robot.

Each light sensor is directed to a fixed direction with $60^{\circ}$ difference between neighbouring neighbouring sensors and detects light coming only from that direction. A wire bundle is attached on top of the hexagonal body to exchange data between the robot and a PC. The wire does not intercept robot locomotion as it hangs from the wall.

To use the Physarum circuit in a robot controller, a bi-directional interface is required for its interaction with the robot. We implemented both directions with optical interfaces mediated by a PC (Fig. 2D).

\section{Cell-to-Robot interface}

A Physarum circuit in a dark chamber is constantly exposed to orange light from the bottom to monitor its activity with a CCD camera. The filtered light contains only the spectral region near $600 \mathrm{~nm}$ to which the cell does not respond. Snapshots of the camera image are saved every two seconds. They are used to calculate the relative thickness of each Physarum oscillator based on the fact that transmitted light through a Physarum circuit is inversely proportional to its thickness. The thickness data from each Physarum oscillator is then converted to a motion for the corresponding leg.

\section{Robot-to-Cell interface}

If a sensor on the robot detects a light signal in the environment, a strong white spotlight shines on the corresponding Physarum oscillator and keeps illuminating it as long as the sensor receives light signals. Unlike the bandpassfiltered light from the bottom, white light does affect the thickness oscillation of the plasmodium [17]. In particular, it triggers changes in oscillation pattern of the Physarum circuit and consequently changes the robot locomotion pattern.

\section{Results}

Without stimulus the thickness oscillation frequency of the Physarum circuit is approximately $0.01 \mathrm{~Hz}$ (100 seconds per period). This value agrees with the literature (e.g. [18]), thus it can be regarded as the inherent frequency of a Physarum circuit. In the presence of light stimli, we observed several reactions. Figure 3 shows two typical reactions of a Physarum oscillator: When a white light stimulus is applied to the Physarum oscillator, the thickness of the oscillator decreased until the stimulus was removed (Fig. 3A), and after some delay the amplitude of oscillation became lower (Fig. 3B). At the same time, 
typically some of the unexposed oscillators increase in thickness and amplitude during the light stimulus.

Based on these observations, an update rule for robot legs has been defined as follows:

$$
\begin{aligned}
& L_{i}(t)= {\left[L_{i}(t-1)+\alpha A_{i}(t) T_{i}(t)\right](\bmod 255) } \\
& A_{i}(t)=d_{i}(t)-d_{i}(t-\tau) \\
& T_{i}(t)=\frac{\sum_{n=0}^{99}((t-n)-\bar{t})\left(d_{i}(t-n)-\overline{d_{i}(t)}\right)}{\sum_{n=0}^{99}((t-n)-\bar{t})^{2}}
\end{aligned}
$$

In the above euqatioins, $L_{i}(t)$ is the position of $i$-th robot leg, taking discrete values from 0 to 255, and $d_{i}(t)$ is the relative thickness of $i$-th Physarum oscillator at time $t . A_{i}(t)$ and $T_{i}(t)$ are an amplitude and a trend of $d_{i}(t)$, respectively. $\bar{t}$ and $\overline{d_{i}(t)}$ are the mean of $(t-n)$ and $d_{i}(t-n)$ with $n=0,1, \cdots, 99 . \alpha$ is the scaling parameter and $\tau$ is the time delay parameter. The above equations represent the motion of each robot leg is determined by thickness changes of the corresponding Physarum oscillator in the star-shaped circuit.

When sensors on the robot detect light signals from a single light source, this update rule induces anti-phase motion between the leg on the side of the sensor and opposing legs. This is the case because stimulated oscillators result in smaller $A_{i}(t)$ and negative $T_{i}(t)$ whereas other oscillators have larger $A_{i}(t)$ and positive $T_{i}(t)$. Eventually this will lead to the robot moving away from the light source.

\subsection{The second generation of the $\Phi$-bot: On-board cellular controller}

Although the first generation robot design allows the cell to control a robot, there are several drawbacks. From a practical perspective, the need to keep the plasmodium moist but avoid condensation obstructing the camera view is a challenge. It also is desirable to reduce the size of the interface to a scale which makes an autonomous robot feasible. Our recent work addresses these issues. To integrate the plasmodium into an autonomous robot it was therefore desirable to explore other technologies for monitoring the activity of the plasmodium. A custom circuit board for electrical impedance spectroscopy (EIS) has been developed [19] and mounted on a small wheeled robot platform [20].

Figure 4A shows the new setup of the bio-hybrid robotic system. The system consists of four components to be described below: a Physarum chip (Fig. 4B), an EIS board, a small gumstix computer, and a wheeled robot base. In this configuration the cell's oscillations are read through impedance measurements and mapped onto the wheel motion of the robot base. The current implementation 
of the un-tethered robot still lacks the interface between the cell and sensors on the robot, i.e., the robot is driven by the cell's oscillating pattern without any feedback to the cell.

\section{The Physarum chip}

The Physarum chip is a small printed circuit board (PCB) containing two plasmodial cells, one to control the robot and one for reference. To maintain the moisture required by the cell, the PCB is covered on one side with an $1.5 \%$ agar gel block, and on the other side with a sheet of the gas-permeable elastomer polydimethylsiloxane (PDMS). The copper side of the PCB with its patterned electrodes faces the agar gel and is insulated from it with laminate. The stack of PDMS-PCB-Agar is clamped with a plexiglass frame. This assembly, referred to as "Physarum chip", completely encloses the plasmodial cell and provides the necessary humidity and adequate oxygen supply to keep the cell active for more than 8 hours. Each plasmodium is confined in a dumbbell-shaped cut-out of the PCB sheet, as shown in Fig. 4B. The dumbbell-shape design, two 1.6 $\mathrm{mm}$ diameter circular holes at a centre-distance of $2.5 \mathrm{~mm}$ connected by a 0.4 $\mathrm{mm}$ wide channel, follows [21, 22] who studied the oscillation patterns of the plasmodium confined to this shape. The PCB sheet is equipped with a total of eight pairs of electrodes for the two plasmodia samples, two electrode pairs for each well (Fig. 4B).

\section{The EIS board}

The impedance measurement circuitry (EIS board) allows for monitoring of the plasmodium's activity using the electrical impedance spectroscopy technique. Impedance spectroscopy is a high-speed, non-invasive and label-free technique used to characterise the dielectric properties of biological tissues and cells [23]. We employ this technique to monitor the oscillation activity of the plasmodium by measuring the impedance between two local parts of the cell. If a lower frequency AC signal (around $100 \mathrm{kHz}$ ) is applied to a Physarum cell, a measured impedance value represents the size of the cell. Accordingly, the temporal trace of the impedance values corresponds to thickness changes of the plasmodium cell. The board incorporates two multiplexers which allows to measure impedance between arbitrary two electrodes on the Physarum chip (Fig. 4B). In the robot control experiment described below, impedances of the two plasmodia at all eight electrode positions are measured once per second.

\section{The gumstix computer}

A tiny computer on which a customised Linux kernel has been installed (www . gumstix.com) mediates between the EIS board and the robot base. The gumstix computer configures the board to retrieve the impedance measurements and saves the data in flash memory. After a signal processing of the measurement data, the computer sends commands to a microcontroller in the wheeled robot base via GPIO (General Purpose Input Output) ports.

\section{The wheeled robot base}

A minimalist design of the robot is adopted from the Braitenberg vehicles [24]. It allows for the Physarum chip, the EIS board, and the gumstix computer 
to be mounted, and accommodates the necessary power supplies for the EIS board and the gumstix computer. The base has its own microcontroller that translates simple commands (forward, left, right) from the gumstix computer to the drive level of the stepper motors.

\section{Cell-to-Robot interface}

Although the second generation of the $\Phi$-bot still lacks a feedback interface from the robot to the Physarum chip like its previous generation, we first ran simple experiments to demonstrate how signals from the cell can be used to drive the robot in the new $\Phi$-bot.

The experiments were run on a $1 \mathrm{~m}$ diameter round table. The robot is equipped with two infra-red proximity switches and ignores forward commands if one of these switches detects an edge of the table. This effectively constrains the robot to the area of the table that serves as arena for experiments. Position and direction of the robot are tracked by an Ethernet camera mounted above the table using an illuminated target pattern on top of the robot.

A two-step process converts the measured impedance signals into drive commands for the robot base: signal processing to recover the oscillation state of the cell from the impedance measurements, and mapping of the cells oscillation state into actuator commands. First, the moving average over 15 samples $(\approx$ 15 seconds) of recorded data is calculated to reduce noise in the impedance measurements. At present the circuitry on the robot has not been optimised to reduce noise, but the signals are strong enough that the simple moving average filter works sufficiently well for our purpose. Next a differential of the averaged signal is computed by subtracting the $15 \mathrm{~s}$ delayed signal to remove the long-term trend of the signals [17]. After the signal processing, a command to drive the robot is determined according to the phase relationship between the differential signals from both wells. It is known that the plasmodium, if confined to the dumbbell shape, show in-phase and out-of-phase oscillation patterns between the two wells [22]. Based on this observation, we introduced a simple mapping from oscillations to robot movement: If the two wells are in phase (synchronised mode), the robot takes a random turn. If they are out of phase (phase delayed mode), then it moves straight. The phase relationship is classified by the following simple rule: If the signs of the two differential signals are equal (both oscillations are increasing or both are decreasing), the oscillation state is classified as synchronised mode. If the signs of the differential signals differ, the oscillation state is classified as phase delayed mode. The former is mapped into a random choice of either a "left" or a "right" command, the latter is mapped into a "forward" command. The whole conversion cycle is performed once per second and commands for the robot's actuators are issued accordingly. The update cycle from impedance measurement to change of robot behaviour is once per second.

Fig. 5A and B show signals from two consecutive time periods of a single experiment. The two curves show the magnitude of the impedance from the left and right circular areas (wells) of the dumbbell shaped plasmodium after a noise filter has been applied. 
The trajectories of the robot that results from this mapping are shown in Fig. 5C and D. During the time period shown in panel A of Fig. 5, the oscillations of the two parts of the plasmodium cell are predominately synchronised and accordingly the robots trajectory shows many random pivot turns (Fig. 5C). On the other hand, in the period shown in panels B and D, the robot runs straight more often because the oscillation pattern switched to an out-of-phase mode about midway through the period shown in (Fig. 5B).

Since the current implementation of the $\Phi$-bot has only a uni-directional interface from the plasmodium to the robot, the next required step to complete the second generation of $\Phi$-bot is the implementation of the converse interface from the robot to the cell, i.e., inputs to the plasmodium. This can be achieved by illuminating the cell with white light from suitable LEDs according to signals from sensors on the robot. So far we ran preliminary experiments of white light stimulation to the Physarum chip and confirmed the cell shows similar reactions against the stimuli as the Physarum circuit of the first generation $\Phi$ bot (Fig. 6). Although this part of the interface is still under implementation, our investigations indicate the feasibility of integrating a living cell into the controller of an autonomous robot.

\section{Computation, Control, and Coordination in the $\Phi$-Bot: Material for a Theory of Bounded Computability}

The development of bio-hybrid robots, such as the $\Phi$-bot, is hampered by the fact that available information-theoretic tools in general ignore how nature directly exploits the manifold physical characteristics of its computing substrates. By "information theory" is meant here, not just Shannon's statistical theory of communication [25], but the structural science that constructs mathematical models of the form, meaning, and use of information and applies them to empirical phenomena. In the engineering context of artificial life and robotics, information theory must systematically develop a theory of bounded computability in which generic traits of information-processing systems are related, not with general time and space bounds (as in the theory of computational complexity [26]), but with specific physico-chemical constraints on the realisation of such systems in different classes of computational media (analogously to the theory of bounded rationality [27]). This empirical diversification of information theory would allow the engineering of unconventional computers to utilise empirical knowledge of naturally evolved systems more efficiently since the requirements for particular computational tasks could then be stated directly in terms of physical specifications of computational media [28].

In a theory about possible relations between material media and computational functions of physical systems, the concept of information must integrate the distinction between the behavioural structure of a system, its functional 
structure, and the structure of its material medium. Information is not a concrete entity that can be localised in a particular part of a system; it is an abstract structure that covers the complex systemic interplay of matter, function, and behaviour. Basic information-theoretic concepts that are general enough to describe this interplay for a spectrum of systems as broad as possible, are the concepts of syntax, semantics, and pragmatics [29]. They are roughly defined by the philosopher Charles Morris as follows (here "semiotic" should be understood as synonymous with "information theory", and signs be considered fundamental units of information):

Pragmatics is that portion of semiotic which deals with the origin, uses, and effects of signs within the behavior in which they occur; semantics deals with the signification of signs in all modes of signifying; syntactics deals with combinations of signs without regard for their specific significations or their relation to the behavior in which they occur.

When so conceived, pragmatics, semantics, syntactics are all interpretable within a behaviorally oriented semiotic, syntactics studying the ways in which signs are combined, semantics studying the signification of signs, and so the interpretant behavior without which there is no signification, pragmatics studying the origin, uses, and effects of signs within the total behavior of the interpretation of signs. [30]

Syntax, semantics, and pragmatics are, thus, not simply iuxtaposed subdisciplines that deal with isolated aspects of signs. Syntactic, semantic, and pragmatic properties of signs are related hierarchically to each other, with pragmatics forming the base since it analyses the overall structure of any behaviour in which signs are involved. Semantics and syntax abstract, to different degrees, from the object of pragmatics: semantics focusses on specifically interpretative behaviour, and syntax puts brackets around signification and analyses all possible methods by which signs can be combined with each other.

In a theory of bounded computability, the general definitions of syntax, semantics, and pragmatics must abstract from any particular class of systems, so that the information-theoretic perspective can be applied, not only to human beings, but also to other living systems, to artefacts, and to engineered hybrids, such as the $\Phi$-bot. Syntax, semantics, and pragmatics, thus, denote different ways of structurally representing any kind of information-processing system from a unified point of view, which, nevertheless, should allow for the differentiation between types of computational media.

Though pragmatics is the most fundamental representation of information, the analysis of the $\Phi$-bot in terms of bounded computability shall start with syntax, since - from a general engineering point of view on possible information processing systems - semantics can most easily be described as the study of code-related boundary conditions that must be met by how signs can combine with each other in an information processing system, and pragmatics as the study of behavioural boundary conditions that must be met by how signs 
can signify something in an information processing system. Applied to the $\Phi$ bot, syntax, semantics, and pragmatics shall each be characterised by a topical "keyword": computation, control, and coordination, respectively. This suggests using "Co ${ }^{3}$ approach" as a shorthand title of the following remarks on bounded computability.

\subsection{Computation and the Syntactic Efficiency and Relia- bility of Computational Media}

Information can be represented syntactically by the material structure of a physical system (e.g., a Physarum cell). The spatio-temporal organisation of the material components of a system is then regarded as an actualisation of the syntactic structure of information in a physical medium. The material structure of the medium actually stands for the syntactic structure of information that is constituted by the set of relations of its elements. The dynamics of selforganisation of the physical medium (e.g., the oscillations of the plasmodium) drives the processing of the syntactic representation of information, but does not require a specific information-theoretic explanation.

Important criteria for classifying computational media from a syntactic perspective are how efficient and reliable these media are in processing syntactic representations of information. Yet to describe a system from the perspective of its efficiency and reliability, presupposes the definition of a purpose that the system shall fulfil. Most generally, this is the purpose of computing a function in the mathematical sense, i.e., to compute, given certain input signals, certain output signals that can then be used for a particular task (e.g., as control signals for the actuators of the $\Phi$-bot). Considering a physical system as a computational medium, any of its physical properties acquires a particular objectifiable function in an engineering sense, namely what it contributes to the computation of a function in the mathematical sense. The syntax of a computational medium, seen from a mathematical perspective, can be categorised by the class of mathematical functions it can compute; this might be done in analogy to the classification of abstract automata [31]. Viewed from an engineering point of view, the syntax of a computational medium can be classified by the efficiency and reliability in which it actually computes the mathematical function it shall compute.

\subsection{Control and the Semantic Generality of Computa- tional Media}

By distinguishing between the mathematical sense and the engineering sense of function, the first step towards the semantic perspective on physical systems as information-processing media has already been taken. Information can be represented semantically by the functional structure of a physical system in the engineering sense. The causal order between the material components of

a system is then regarded as an implementation of the semantic structure of 
information by a physical medium. Yet semantics is not coextensive with functionality at large, it is code-based functionality. A code, such as the Morse code and the genetic code, connects two syntactic structures with each other. It is a mapping that relates, in case of encoding, each of the possible syntactic elements of a message to a possible element of a signal and, in case of decoding, each of the possible syntactic elements of a signal to a possible element of a message [32]. It is of great importance to emphasise that the semantic relation between signal and message is contingent, which means that it can be chosen by the engineer from a set of possible mappings all of which are compatible with the natural laws that govern the causal order in the computational medium.

The dynamics of self-organisation of the physical medium implements the semantic structure of information by encoding and decoding syntactic structures in physical processes. From the perspective of semantics, it is necessary to interpret the present state of (a part of) a system as encoding the future state (of another part) of it.

Where is information represented semantically in the interaction loop of the $\Phi$-bot and its environment (see Fig. 7)? First and foremost, in the code-based functional structure of the artificial control. Encoding happens when stimuli from the robotic light sensors are transduced to white light signals for the cell. Decoding occurs when amplitude signals calculated from measured data of the plasmodium oscillators are processed by the update rule to alter the motion of the robot actuators. The update rule acts as a decoding device that semantically relates a syntactic representation of information (plasmodium oscillation signals) to another syntactic representation of information (robot motor signals). All in all, four syntactic representations of information (external light signals, white light signals for the plasmodium, oscillation signals from the plasmodium, and robot motor signals) are related by two semantic representations of information (namely, the code used for encoding the light sensor data into white light signals and the code used for decoding the oscillation data into motor signals). Inbetween, the plasmodium connects the two semantic representations by bounded computation.

It is the semantic structure of the robot control device (in short, its control semantics) that gives a particular signification to certain syntactic substructures in the Physarum cell. Thereby, the plasmodium is regarded as computing a mathematical function and, by doing so, fulfils an engineered function in the $\Phi$-bot. The control semantics is a necessary condition for the computational use of the syntactic structure of the Physarum plasmodium. In code-based mutual constraining, the behavioural changes of the interacting syntactic structures are induced by the rules of the control semantics. Those semantic rules can be described, or implemented, without having a full-fledged theory about the internal workings of the systems involved since the observation of their behaviour suffices to determine the syntactic input into the code.

The set of possible messages that the control semantics, as given by engineered or organic codes [33], can distinguish, is an important semantic criterion for classifying computational media. It measures how general the codes used in a medium to implement semantic representations of information are, i.e., to 
which degree the codes are able to differentiate between possible messages under given boundary conditions. From the perspective of the computational medium, the control semantics acts as a behaviour amplifier. The microscopic behaviour of, e.g., a Physarum cell is amplified to the macroscopic behaviour of a robot by semantic means (analogously to the hierarchical mode of biological information processing [1]). This suggests to think about the generality of codes, i.e., about the degree to which they are able to differentiate between possible messages in relation to given pragmatic boundary conditions, in terms of the graininess of behaviours between which the codes can differentiate. The finer the behavioural differences of the Physarum cell that a code can semantically represent are, the more general the code is in respect to this particular control setting.

\subsection{Coordination and the Pragmatic Versatility of Com- putational Media}

By describing the capability of the control semantics to make distinctions between messages, i.e., the generality of the implemented codes, in terms of behaviour, the first step towards the pragmatic perspective on physical systems as information-processing media has already been taken. The very idea of control is to intervene in a process that, by ensuing interaction, shall produce a particular result in the influenced process [34]. This process is the real-life function that the system in which the controller works, shall fulfil in a certain class of environments. In case of both the $\Phi$-bot and the Physarum cell, a particular type of behaviour as regards light sources is to be shown. The $\Phi$-bot does so by an engineered control semantics, the Physarum cell by an evolved one.

Information can be represented pragmatically by the behavioural structure of a physical system. The pattern of interaction between the system and its environment is then regarded as an effectuation of the pragmatic structure of information through the agency of a physical medium. The pragmatic structure is constituted by transformations of boundary conditions on coding. When is a message selected for being encoded, when is a signal decoded, and how does the code originate? Generalising the idea that information is constituted pragmatically by the effect of a signal on its receiver [35], the definition of the pragmatic structure of information involves at least two syntactic orders and one semantic mapping.

The dynamics of self-organisation of the physical medium changes internal and external conditions of information processing in the system. From the perspective of pragmatics, it is necessary to interact with the present behaviour of a system in order to let its dynamics lead it to a particular future behaviour.

Where is information represented pragmatically in the interaction loop of the $\Phi$-bot and its environment? First, the behaviour of the robot that results from decoding plasmodium oscillation signals into robot motor signals, changes the boundary conditions on encoding since the effects of the robot's activity on the environment are perceived by the robot's light sensors whose data is then encoded into white light signals for the cell. Second, the plasmodium behaves according to its own dynamics in its direct environment, i.e. in the artificial 
control. This environment receives the behaviour of the plasmodium in form of oscillation data that is decoded into robot motor signals. The pragmatic interaction of the plasmodium with its engineered environment is, thus, semantically represented in the very same environment and then pragmatically represented by the behaviour of the robot in its real-world environment. The control semantics as given by the two codes described in section 4.2 connects the relation of the robot to its real-world environment with the relation of the plasmodium to its artificial environment. The control semantics, thus, maps two different pragmatic representations of information to each other, namely the behaviour of the plasmodium and the behaviour of the robot.

The codes used in the $\Phi$-bot for encoding external stimuli and for decoding internally collected measurement data, are pragmatically connected by the behaviour of the Physarum cell, i.e. by how it measurably reacts to the encoded stimuli. To encode information means in pragmatic respect that the control device sets the boundary conditions on how the plasmodium processes syntactic representations of information. To decode information means that the control device semantically represents the pragmatic results of information processing by the Physarum cell.

How can this interplay of semantics and pragmatics be described more generally? From a pragmatic perspective, the control semantics can be seen as if it is a signalling convention between two independent information-processing systems that has arisen from a proper equilibrium in the coordination of the behaviours of both systems, here of a Physarum plasmodium and a robot, the latter representing the designer's intentions. By 'proper coordination equilibrium' is meant a combination of behaviours in which any agent would have a disadvantage if any one agent had acted otherwise [36]. To put it more realistically, the robot's designer has selected both systems according to their respective capacity to cooperatively realize, in a stable way, the intended real-life function of the $\Phi$-bot by means of the signalling convention that is called 'control semantics'.

The key engineering issue here is how we can exploit the cell's self-organising dynamics to achieve a fully autonomous robot. Arguably, biological cells outperform conventional autonomous robots in many features by exploiting pragmatic versatility, i.e. the high degree to which the cells are able to adapt their behaviour to different environments. An important criterion for classifying computational media from a pragmatic perspective, thus, is how versatile media are in effectuating transformations of the system's behaviour under changing boundary conditions, i.e., to which degree the behaviour of the system is able to adapt itself to different environments. Pragmatic versatility depends on the diversity of environments in which the cooperation of the computational medium and the robot is actualised successfully. For the $\Phi$-bot, the interplay of semantics and pragmatics via its controller is as important as the syntactic efficiency of the Physarum cell, since this interplay is the means by which the pragmatic versatility of the plasmodium is also detectable in the behaviour of the $\Phi$-bot.

Coordination between the plasmodium and the robot is relatively easy in case of the $\Phi$-bot since there does not exist a coordination problem with more than one proper coordination equilibrium. If the coordination of a computa- 
tional medium and a robot has been stabilised by a control semantics in a situation with only one coordination equilibrium, the behaviour of the robot in this situation can, in principle, be anticipated by an external observer. The engineering of control semantics will become more difficult if computational media showing a more unpredictable behaviour than Physarum plasmodium, shall be used to control robots whose intended behaviour must be modelled by more complex utility functions than it is necessary for the $\Phi$-bot. Even a Physarum cell sometimes shows a behaviour that goes in this direction.

Several researchers have observed that the plasmodium is able to spontaneously change its behaviour pattern against external stimuli to overcome unfavourable conditions [37, 38, 39]. For example, Takamatsu found that if the plasmodium is entrained to oscillate at a fixed frequency by external periodicallychanging stimulus, it spontaneously deviates from the frequency after a certain period even if the stimulus is maintained. She speculated that such spontaneous change might stem from multistability or chaotic behaviour of the plasmodium's dynamics and may contribute to the diversity of behavioural modes of the plasmodium, such as food-searching mode and feeding mode [39].

Although physiological mechanisms underlying such behaviour are yet to be investigated, these observations point to richness of the cell's internal dynamics. However, they also point to the lack of theory about relations between the dynamics of physico-chemical material structures and their use as computational media. The conventional computing paradigm assumes perfection of each part in a system. It is, therefore, inadequate when we want to harness the pragmatic versatility of the plasmodium, which results from the richness of its self-organised processing of syntactic representations of information, by a control semantics that allows the robot to adapt its behaviour to a real-world environment.

\section{Conclusion}

In this chapter, we reviewed our cellular robot control approach toward adaptive autonomous robots, followed by a first outline of a theoretical approach to biohybrid computing.

We have shown that properties of Physarum cells can meet the requirements for the adaptive behaviour generation in engineered systems and how those properties are employed for the control of autonomous robots. Although this work is still ongoing and many problems remain to be solved, we can expect that the use of biological cells will provide several features difficult to achieve with conventional technologies, such as self-reconfiguration and self-repair capabilities. A tight integration of cells with existing technologies will require fine control of the micro-environment of the cells. Lab-on-chip technogy can be employed to achieve this [40]. In current work, our laboratory investigates, for example, whether the cellular controller can be "swtiched off" and stored for a long time by keeping it in a dry dormant state and, when needed, easily "powered on" by supplying moisture through microfluidics. 
Because unconventional computing devices are radically different from conventional computers, it is inevitable to develop a new theory to capture the informational significance of physical properties of novel computational media. Bounded computability as outlined by the $\mathrm{Co}^{3}$ approach must be subjected to further discussion and needs to be developed into a full-fledged theory. This presupposes that formal approaches to pragmatic, semantic, and syntactic representations of information must be integrated into a general concept of computability under different types of physical constraints. This is, of course, a very challenging task: it amounts to regarding complex adaptive systems as models of information theory (here, 'model' is to be understood in the sense of formal semantics, the mathematical discipline describing the relation between a theory and objects that fulfil this theory). On an even broader horizon, the theory of bounded computability and the engineering of bio-hybrid devices together may make an essential contribution to our general understanding of informational behaviour shown by any kind of adaptive system.

A most important conceptual precondition for reaching such a general understanding of informational behaviour is a consequent relativism in thinking about information. What is to be considered a syntactic, semantic, or pragmatic representation of information, depends on the interaction between the described and the describing system. For example, material features of computational media, such as the plasmodium in the $\Phi$-bot, appear, from the perspective of a code-based controller, primarily as constraints on the processing of syntactic representations of information. Information processing by the computational medium has, of course, also internal semantic and pragmatic aspects. There exist, e.g., semantic representations of information in the Physarum cell, such as the organic code that structures the expression of genetic information. Yet from the perspective of the controller those cell-internal aspects are to be considered just as constraints on the efficiency and reliability of processing syntactic representations of information that are actualised in the cell's environment. Therefore, the syntactic effectiveness and reliability of the computational medium show themselves, to the controller, in the pragmatic versatility of the medium, i.e., the degree to which its behaviour is able to adapt itself to changing boundary conditions that bear information syntactically. To describe such complex relations between syntax, semantics, and pragmatics, the theory of bounded computability must assume a relativistic attitude towards information.

\section{References}

[1] M. Conrad. The importance of molecular hierarchy in information preocessing. In C. H. Waddington, editor, Towards a Theoreritcal Biology, volume 4, pages 222-228. Edinburgh University Press, 1972.

[2] K.-P. Zauner. Molecular information technology. Critical Reviews in Solid State and Material Sciences, 30(1):33-69, 2005.

[3] A. Adamatzky, B.D.L. Costello, and T. Asai. Reaction-Diffusion Computers. Elsevier Science Inc., New York, NY, USA, 2005. 
[4] K. E. Wohlfarth-Bottermann. Oscillatory contraction activity in physarum. The Journal of Experimental Biology, 81:15-32, 1979.

[5] Z. Hejnowicz and K. E. Wohlfarth-Bottermann. Propagated waves induced by gradients of physiological factors within plasmodia of Physarum polycephalum. Planta, 150:144152,1980

[6] K. Matsumoto, T. Ueda, and Y. Kobatake. Propagation of phase wave in relation to tactic responses by the plasmodium of Physarum polycephalum. Journal of Theoretical Biology, 122:339-345, 1986.

[7] H. Tanaka, H. Yoshimura, Y. Miyake, J. Imaizumi, K. Nagayama, and H. Shimizu. Information processing of Physarum polycephalum studied by micro-thermography. Protoplasma, 138:98-104, 1987.

[8] K. Matsumoto, T. Ueda, and Y. Kobatake. Reversal of thermotaxis with oscillatory stimulation in the plasmodium of Physarum polycephalum. Journal of Theoretical Biology, 131:175-182, 1988.

[9] H. Miura and M. Yano. A model of organization of size invariant positional information in taxis of Physarum plasmodium. Progress of Theoretical Physics, 100(2):235-251, 1998.

[10] Y. Miyake, S. Tabata, H. Murakami, M. Yano, and H. Shimizu. Environmental-dependent self-organization of positional information field in chemotaxis of Physarum plasmodium. Journal of Theoretical Biology, 178:341-353, 1996.

[11] A. Gierer and H. Meinhardt. Theory of biological pattern formation. Kybernetik, 12:3039, 1972.

[12] A. Takamatsu and T. Fujii. Construction of a living coupled oscillator system of plasmodial slime mold by a microfabricated structure. Sensors Update, 10(1):33-46, January 2002.

[13] A. Takamatsu, R. Tanaka, H. Yamada, T. Nakagaki, T. Fujii, and I. Endo. Spatiotemporal symmetry in rings of coupled biological oscillators of physarum plasmodial slime mold. Phys. Rev. Lett., 87(7):078102, Jul 2001.

[14] T. Nakagaki, R. Kobayashi, Y. Nishiura, and T. Ueda. Obtaining multiple separate food sources: behavioural intelligence in the Physarum plasmodium. R. Soc. Proc.: Biol. Sci., 271(1554):2305-2310, 2004.

[15] A. Adamatzky. Physarum machine: Implementation of a Kolmogorov-Uspensky machine on a biological substrate. Parallel Processing Letters, 17(4):455-467, 2007.

[16] T. Saigusa, A. Tero, T. Nakagaki, and Y. Kuramoto. Amoebae anticipate periodic events. Physical Review Letters, 100(1):018101, 2008.

[17] T. Nakagaki, S. Uemura, Y. Kakiuchi, and T. Ueda. Action spectrum for sporulation and photoavoidance in the plasmodium of Physarum polycephalum, as modified differentially by temperature and starvation. Photochemistry and Photobiology, 64(5):859-862, 1996.

[18] K. E. Wohlfarth-Botterman. Oscillating contractions in protoplasmic strands of physarum: Simultaneous tensiometry of logitudinal and radial rhythms, periodicity analysis and temperature dependence. Journal of Experimental Biology, 67:49-59, 1977.

[19] P. Macey. Impedance spectroscopy based interfacing with a living cell for biosensors and bio-coporcessors. Part III Project Report, School of Electronics and Computer Science, University of Southampton, May 2007.

[20] G. Jones. Robotic platform for molecular controlled robots. Part III Project Report, School of Electronics and Computer Science, University of Southampton, May 2006. 
[21] A. Takamatsu, T. Fujii, and I. Endo. Control of interaction strength in a network of the true slime mold by a microfabricated structure. BioSystems, 55:33-38, 2000.

[22] A. Takamatsu, T. Fujii, H. Yokota, K. Hosokawa, T. Higuchi, and I. Endo. Controlling the geometry and the coupling strength of the oscillator system in plasmodium of Physarum polycephalum by microfabricated structure. Protoplasma, 210:164-171, 2000.

[23] H. G. L. Coster, T. C. Chilcott, and C. F. Coster. Impedance spectroscopy of interfaces, membranes and ultrastructures. Bioelectrochemistry and Bioenergetics, 40:79-98, 1996.

[24] V. Braitenberg. Vehicles: Experiments in Synthetic Psychology. MIT Press, Cambridge, MA, 1984.

[25] C. Shannon and W. Weaver. Mathematical Theory of Communication. University of Illinois Press, 1949.

[26] C. M. Papadimitriou. Computational complexity. Addison-Wesley, Reading, Massachusetts, 1994.

[27] H.A. Simon. Models of Bounded Rationality, three volumes. MIT Press, Cambridge, Massachusetts, 1982/1997.

[28] S. Tsuda, K.-P. Zauner, and Y.-P. Gunji. Computing substrates and life. In S. Artmann and P. Dittrich, editors, Explorations in the Complexity of Possible Life: Abstracting and Synthesizing the Principles of Living Systems, Proceedings of the 7th German Workshop on Artificial Life, pages 39-49, Jena, Germany, July 26-28 2006. IOS Press.

[29] S. Artmann. Biological information. In S. Sarkar and A. Plutynski, editors, A Companion to the Philosophy of Biology, pages 22-39. Malden/MA: Blackwell, 2008.

[30] C. Morris. Writings on the General Theory of Signs. Mouton, Den Haag and Paris, 1971.

[31] J. E. Hopcroft, R. Motwani, and J. D. Ullman. Introduction to Automata Theory, Languages, and Computation. Addison-Wesley, Reading, Massachussetts, third edition, 2007.

[32] T. M. Cover and J. A. Thomas. Elements of Information Theory. Wiley-Interscience, 2nd edition, 2006.

[33] M. Barbieri. Organic Codes: An Introduction to Semantic Biology. Cambridge University Press, Cambridge/England, 2003.

[34] S. Artmann. Basic semiosis as code-based control. Biosemiotics, 2009. (in press).

[35] D.M. MacKay. Information, Mechanism and Meaning. Cambridge, MA: MIT Press, 1969.

[36] D.K. Lewis. Convention: a philosophical study. Harvard University Press, Princeton, New Jersey, first edition, 1968.

[37] M. Aono and M. Hara. Amoeba-based nonequilibrium neurocomputer utilizing fluctuations and instability. In 6th International Conference, UC 2007, volume 4618 of LNCS, pages 41-54, Kingston, Canada, August 13-17 2007. Springer.

[38] S. Nomura. Symbolization of an object and its freedom in biological systems. PhD thesis, Kobe University, 2001.

[39] A. Takamatsu, T. Yamamoto, and T. Fujii. Spontaneous switching of frequency-locking by periodic stimulus in oscillators of plasmodium of the true slime mold. BioSystems, 76:133-140, 2004.

[40] F.D. Revilla, K.-P. Zauner, and H. Morgan. Physarum polycephalum on a chip. In J.-L. Viovy, P. Tabeling, S. Descroix, and L. Malaquin, editors, The proceedings of $\mu$ TAS 2007, volume 2, pages 1089-1091, 2007. 


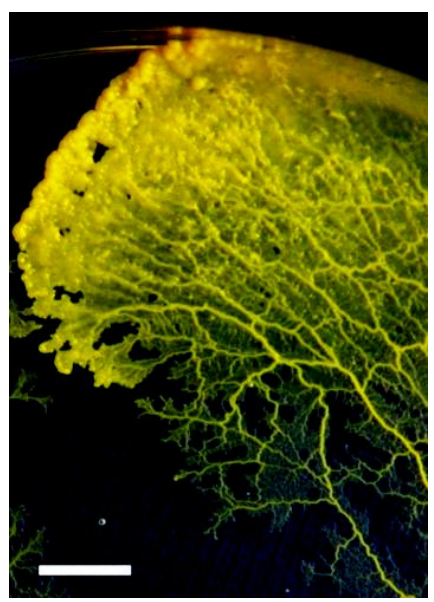

Figure 1: The plasmodium of the true slime mould Physarum polycephalum growing on $1.5 \%$ agar gel. The white bar on the panel indicates $5 \mathrm{~mm}$. 
A

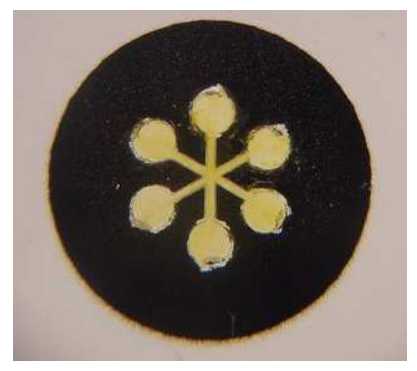

B

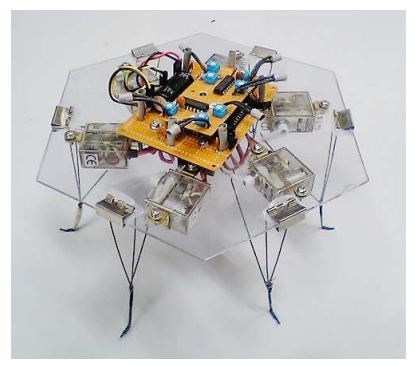

C

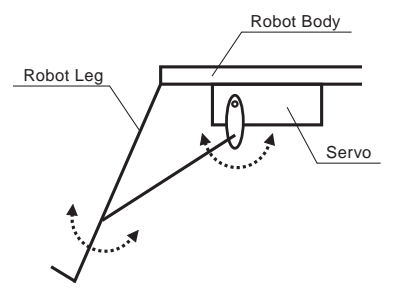

D

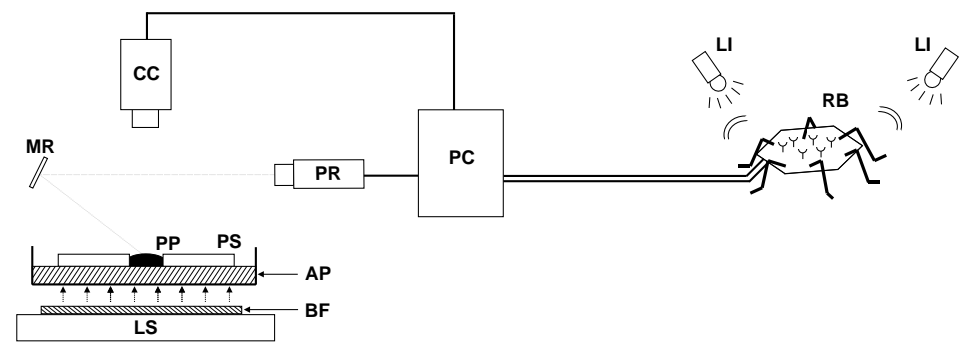

Figure 2: Components for Physarum controlled robot. The shape of the Physarum cell can be controlled by a dry negative mask on a $1.5 \%$ agar gel plate (A). Each Physarum oscillator controls one leg in a hexapod robot (B). Each leg has only one degree of freedom $(\mathrm{C})$ and the combination of six leg motions generates various gate patterns. These two components are interfaced by bidirectional interfaces: Sensors on the robot $(\mathrm{RB})$ detect light inputs $(\mathrm{LI})$ in the environment and transmit signals to the computer $(\mathrm{PC})$. The signals from the sensors are recoded by the PC into a spatial light pattern which is projected as light stimulus with a video projector $(\mathrm{PR})$ via mirror $(\mathrm{MR})$ onto the surface of the plasmodium (PP). Oscillations of the plasmodium (PP), which is patterned on an agar plate (AP) by a plastic sheet (PS), are detected by a CCD camera (CC) as intensity changes in light transmitted from the bandpass filtered (BF) light source (LS). 

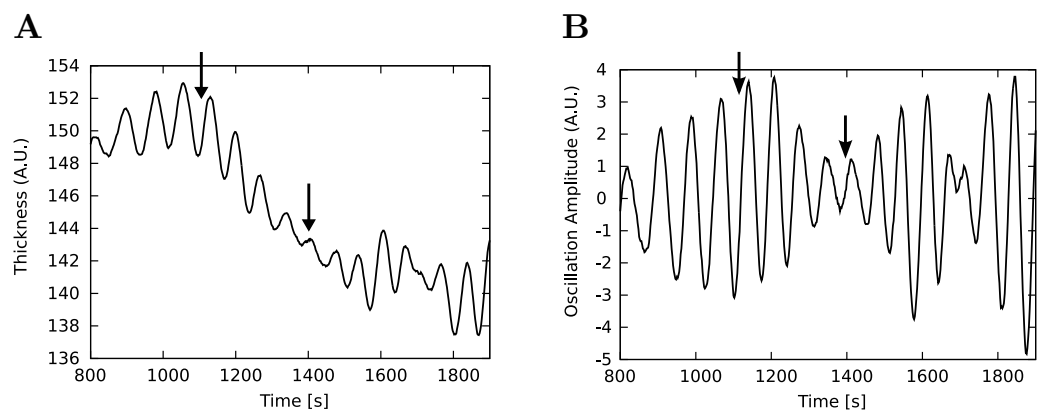

Figure 3: Reactions of a Physarum oscillator to light stimulus. Decrease in thickness of the oscillator is observed (A) along with reduction of the oscillation amplitude (B). Arrows in the graphs indicate start (left) and end (right) of the light exposure.

A

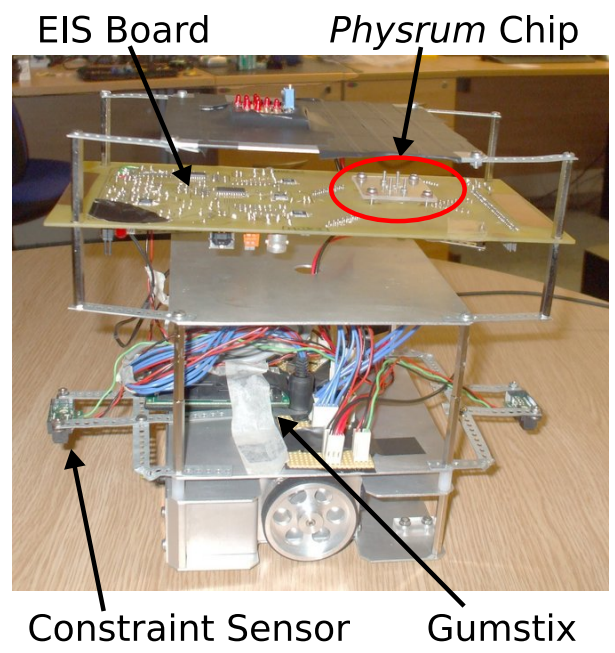

B

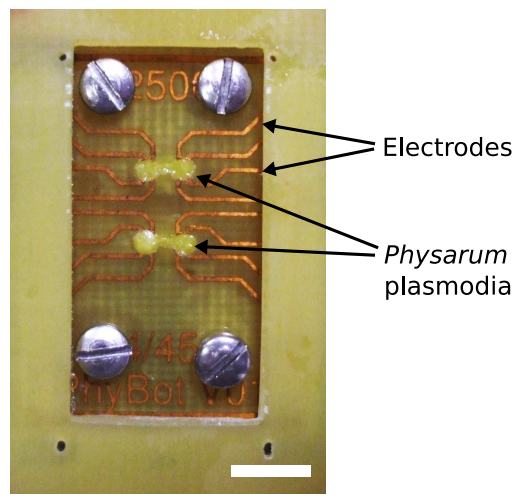

Figure 4: The complete setup of the robotic system (A) driven by the Physarum plasmodium growing in the Physarum chip (B). The white bar in (B) corresponds to $5 \mathrm{~mm}$. 
A

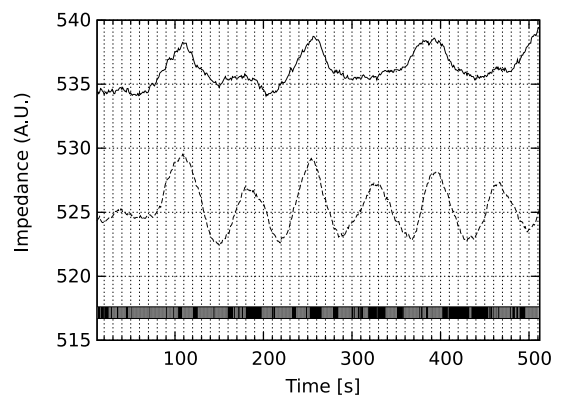

C

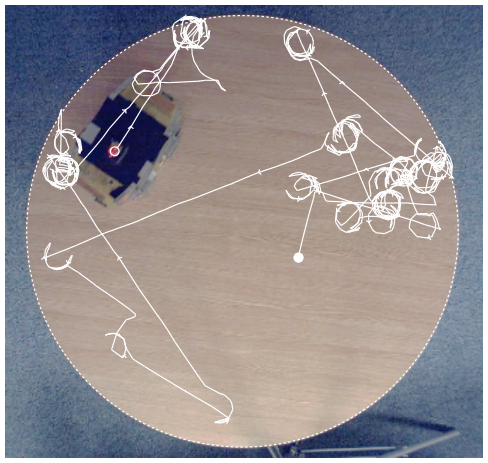

B

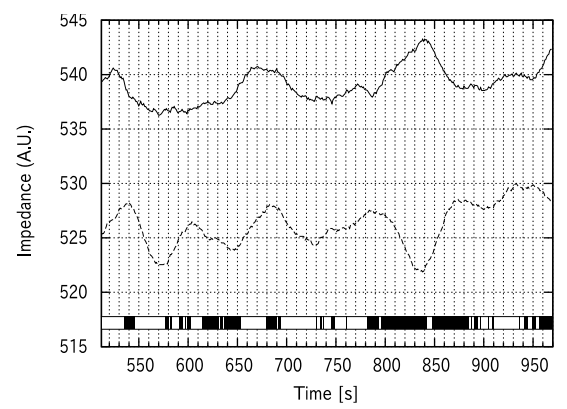

D

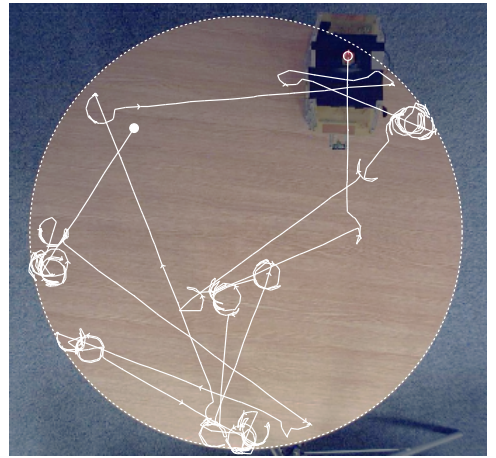

Figure 5: Oscillation of a plasmodium in a Physarum chip and the corresponding trajectories of the robot. The moving-averages of the magnitude of the impedance at $100 \mathrm{k} \mathrm{Hz}$ are plotted for 30-500 s (A) and 500-970 s (B). The solid and dotted curves in the plots correspond to oscillations of the plasmodium from right and left wells, respectively. The phase relationship between two wells are shown in the bottom of the plots as black (in-phase) and white (out-of-phase) vertical lines. The behaviour of the robot is determined according to the phase relationship as traced in panel (C) for 30-500 s and panel (D) 500-970 s. A white solid circle indicates the start and an open circle the end of the trajectory. 


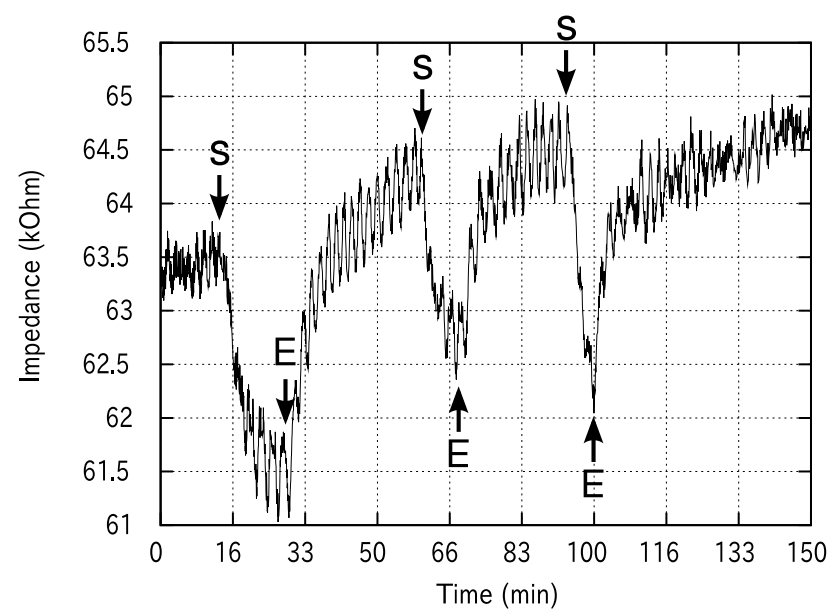

Figure 6: The impedance oscillation of a stimulated well in a Physarum chip. When one of two wells in a Physarum chip is exposed to white light stimulus, it shows sharp decrease in impedance (corresponding to thickness of the cell within the well). S and $\mathrm{E}$ in the graph indicate start and end of light exposures, respectively.

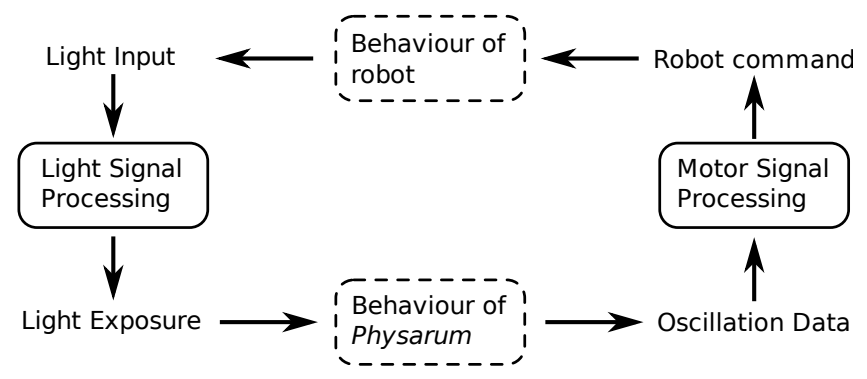

Figure 7: Interaction loop of the $\Phi$-bot. Each part of the diagram corresponds to either syntax (no box), semantics (solid box), or pragmatics (dotted box). 